Mon. Not. R. Astron. Soc. 000, 1-15 (XXX) Printed 15 June $2018 \quad$ (MN LATEX style file v2.2)

\title{
Star formation history of KDG 61 and KDG 64 from spectroscopy and colour-magnitude diagrams ${ }^{\star} \dagger$
}

\author{
Lidia Makarova ${ }^{1,2} \ddagger$, Mina Koleva ${ }^{3,2}$, Dmitry Makarov ${ }^{1,2}$, Philippe Prugniel ${ }^{2}$ \\ ${ }^{1}$ Special Astrophysical Observatory, Nizhniy Arkhyz, Karachai-Cherkessia 369167, Russia \\ Isaac Newton Institute of Chile, SAO Branch, Russia \\ ${ }^{2}$ Université Lyon 1, Villeurbanne, F-69622, France; CRAL, Observatoire de Lyon, St. Genis Laval, F-69561, France ; \\ CNRS, UMR 5574 \\ ${ }^{3}$ Instituto de Astrofísica de Canarias, La Laguna, E-38200 Tenerife, Spain \\ Departamento de Astrofísica, Universidad de La Laguna, E-38205 La Laguna, Tenerife, Spain
}

Accepted 2010 March 25. Received 2010 March 17; in original form 2010 January 22

\begin{abstract}
A study of two dE/dSph members of the nearby M 81 group of galaxies, KDG 61 and UGC $5442=$ KDG 64, has been made. Direct Hubble Space Telescope (HST) Advanced Camera for Surveys (ACS) images and integrated-light spectra of $6 \mathrm{~m}$ telescope of Special Astrophysical Observatory of Russian Academy of Sciences have been used for quantitative star formation history analysis. The spectroscopic and colour-magnitude diagrams analysis gives consistent results. These galaxies appear to be dominated by an old population (12-14 Gyr) of low metallicity $([\mathrm{Fe} / \mathrm{H}] \sim-1.5)$. Stars of ages about 1 to 4 Gyr have been detected in both galaxies. The later population shows marginal metal enrichment. We do not detect any significant radial gradients in age or metallicity in these galaxies. Our radial velocity measurement suggests that the HiI knot on the line-of-sight of KDG 61 is not gravitationally attached to the galaxy.
\end{abstract}

Key words: galaxies: dwarf - galaxies: formation - galaxies: evolution - galaxies: stellar content - galaxies: individual: KDG 61 - galaxies: individual: KDG 64

\section{INTRODUCTION}

Most of the known galaxies are dwarfs. They span mass ranges from a few $10^{9} \mathrm{M}_{\odot}$, (or $\left.\mathrm{M}_{V} \simeq-18 \mathrm{mag} ; 10^{9} \mathrm{~L}_{\odot}\right)$ like NGC 205, to $10^{7} \mathrm{M}_{\odot}\left(\mathrm{M}_{V} \simeq-12 \mathrm{mag} ; 10^{6} \mathrm{~L}_{\odot}\right)$, like Fornax and Sculptor galaxies, satellites of the Milky Way, and probably down to $10^{5} \mathrm{M}_{\odot}\left(\mathrm{M}_{V} \simeq-3 \mathrm{mag} ; 1000 \mathrm{~L}_{\odot}\right)$ for the fainter Milky Way satellites found from the SDSS (Koposov et al. 2009). Dwarfs vary considerably in their gas content and morphology, between dwarf irregular galaxies (dIrr) and dwarf elliptical or spheroidal galaxies (dE/dSph). Environmental effects likely drive the evolution of star forming dwarf galaxies to the quiescent ones after gas removal and/or exhaustion. Among the quiescent objects, the fainter ones are traditionally called $\mathrm{dSph}$ while the more massive are

\footnotetext{
* Based on observations made with the NASA/ESA Hubble Space Telescope, obtained from the data archive at the Space Telescope Science Institute. STScI is operated by the Association of Universities for Research in Astronomy, Inc. under NASA contract NAS 5-26555.

$\dagger$ Based on observations made with the 6m BTA telescope of the Special Astrophysical Observatory, Russian Academy of Sciences. $\ddagger$ E-mail: lidia@sao.ru
}

named dE (diffuse or dwarf ellipticals; e.g. Grebel 1999). The bound is often set at $M_{V} \simeq-14$ mag. However, the physical distinction between these two classes is unclear. Dwarf spheroidals are generally companions of massive galaxies $(20$ of them were identified around the Milky Way and 15 around Andromeda; Irwin et al. 2008) while dEs are found in clusters (1141 of them are listed in the Virgo Cluster Catalogue; Binggeli et al. 1985), but NGC 205, prototype of dEs, is also a companion of M31, and faint $\mathrm{dE} / \mathrm{dSph}$ are now found in nearby clusters (Trentham \& Hodgkin 2002; Mieske et al. 2007; Adami et al. 2007; de Rijcke et al. 2009). The nearby dSphs are often found to be dominated by dark matter (Mateo 1998), while the more massive, dEs are apparently similar to the massive elliptical galaxies: the stellar content of NGC 147, NGC 185 and NGC 205 account for half of their dynamical masses (De Rijcke et al. 2006). Other properties, like the Sérsic index, n, characterizing the shape of the photometric profile, seems to form a continuum over all the mass range. de Rijcke et al. (2009) proposes that the Sérsic index is independent of the luminosity for $\mathrm{M}_{V} \gtrsim-14 \mathrm{mag}$, $n \approx 0.7$, and increases with the luminosity above this limit. The change in the photometric scaling relation may reflect the different nature of dEs and dSphs, but both the mea- 
surement uncertainties and the cosmic dispersion are still large enough to dispute such a dichotomy.

Exploring the mass sequence of dEs/dSphs is a significant step toward the understanding of the different processes of their formation and evolution. Is there a sharp transition of the dark matter content at some intermediate mass? Were all these galaxies formed at the same epoch and are there any systematics in the star formation?

To address these questions, we have studied two of the nearest $\mathrm{dE} / \mathrm{dSph}$ galaxies with intermediate masses, located in the M 81 group at about $3.6 \mathrm{Mpc}$. They are two of the brightest $\mathrm{dE} / \mathrm{dSph}$ in this group: UGC $5442=\mathrm{KDG} 64$ and KDG61. The KDG designations (Karachentseva dwarf galaxies) are from the galaxy catalogue by Karachentseva (1968).

Both objects are situated in the central region of the group. KDG 61 is located 29.5 arcmin South of M 81, i.e. $31 \mathrm{kpc}$ in projected distance. KDG64 is projected 58.2 arcmin South of NGC $3077(61.5 \mathrm{kpc})$ and $97.5 \mathrm{arcmin}$ South-East of M 81 (103 kpc). KDG 61 is the most luminous dSph in the M 81 group and it is one of the closest companions to the M81 galaxy. Johnson et al. (1997) has found a His knot 34 arcsec NE from the centre $(0.6 \mathrm{kpc}$ in projection, position J2000 09:57:07.48+68:35:53.9). This sign of recent star formation and the detection of a Hi cloud led Karachentsev et al. (2000) and Boyce et al. (2001) to classify the galaxy as transitional type, between dIrr and dSph. A globular cluster has been discovered in KDG61 (Sharina et al. 2005) at the projected distance of $0.05 \mathrm{kpc}(3 \mathrm{arcsec})$ from the galaxy's centre. This cluster has an effective radius of $4.7 \mathrm{pc}(0.3 \mathrm{arcsec})$, absolute luminosity $\mathrm{M}_{V}=-7.55$ and a de-reddened colour of $V-I=0.92$. This colour is similar to the rest of the galaxy (see Table 1). In spite of the fact that KDG64 is fainter than KDG61, it has half magnitude higher surface brightness. The nucleus-like object (Binggeli \& Prugniel 1994; Bremnes et al. 1998) is a distant galaxy (Karachentsev et al. 2000; Simien \& Prugniel 2002). No globular cluster was detected by Sharina et al. (2005). The internal kinematics of KDG 64 was studied using long-slit spectroscopy by Simien \& Prugniel (2002).

The absolute luminosity, total colour and other general parameters of the both galaxies are shown in the Table 1. Coordinates are taken from HyperLEDA ${ }^{1}$ (Paturel et al. 2003). Total $B$ and $R$ magnitudes are taken from Bremnes et al. (1998), central surface brightness in $V$ and $(V-I)$ colour are from Sharina et al. (2008). The axial ratio was taken from the 'A Catalog of Neighboring Galaxies' (Karachentsev et al. 2004) and the central velocity dispersion from Prugniel \& Simien (2003). The rest of values are from this work. All magnitudes and colours are corrected for Galactic extinction using the Schlegel et al. (1998) maps.

In Sect. 2 we analyse colour-magnitude diagrams (CMDs) of KDG 61 and KDG 64 derived from HST/ACS images. We measure accurately the distance and find the star formation and metal enrichment history. In Sect. 3 we present the long-slit spectra that we obtained and their analysis using full spectrum fitting. Section 4 discusses the nature of these galaxies and Section 5 gives our conclusions.

\section{DIRECT IMAGES AND STAR FORMATION HISTORY}

\subsection{Observational material and photometry}

Direct images of KDG 61 and KDG 64 were taken from the HST data archive (proposal 9884, PI Taft Armandroff). Deep HST/ACS observations of the two galaxies were made using $F 606 W$ (broadband $V$ ) and $F 814 W$ (broadband $I$ ) filters. The cosmic ray cleaned images were obtained from the STScI data archive and have been processed with the standard ACS pipeline. The images of the two galaxies are shown in Fig. 1 (combined distortion-corrected mosaic images corresponding to an exposure time of 8600s).

The photometry of resolved stars in the galaxies was made with the ACS module of the DOLPHOT package ${ }^{2}$ for crowded field photometry (Dolphin 2002) using the recommended recipe and parameters. Charge transfer efficiency corrections were made according to ACS ISR03-09, and the transformation into the standard $U B V R I$ photometric system were made according to Sirianni et al. (2005). We estimate the uncertainties in the calibration to be 0.05 to 0.10 magnitudes. Note, however, that we used these standard magnitudes for reference only; all our measurements were made in the original ACS photometric system to avoid additional systematic uncertainties.

Only the stars with photometry of good quality were included in the final catalogue, following the recommendations given in the DOLPHOT User's Guide. We have selected stars with signal-to-noise $(\mathrm{S} / \mathrm{N})$ of at least five in both filters, $\chi^{2} \leqslant 5.0$ and $|\operatorname{sharp}| \leqslant 0.3$. The resulting CMDs of the two dSphs are presented in Fig. 2. The CMD of KDG 61 contains 59554 stars and the one of KDG 64 contains 45779 stars.

Artificial star tests is the only accurate way to solve the problems of photometric errors, blending and incompleteness (see, for example, Gallart et al. 1996, Dolphin 2002). These tests were performed for both galaxies using the same reduction procedures. A large library of artificial stars was generated spanning the necessary range of stellar magnitudes and colours so that the distribution of the recovered photometry is adequately sampled. The photometric errors and completeness, represented in Fig. 3, are similar for the both galaxies. The $1 \sigma$ photometric precision is about $0.08 \mathrm{mag}$ at $F 814 W=26$ and $0.18 \mathrm{mag}$ at $F 814 W=27$. The Malmquist bias becomes notable for stars with $F 814 W>27.2$. In $F 606 W$ the $1 \sigma$ photometric precision is about $0.05 \mathrm{mag}$ at $26 \mathrm{mag}$ and about 0.21 at $28 \mathrm{mag}$.

\subsection{Colour-magnitude diagrams}

The CMDs of both KDG61 and KDG64 (Fig. 2) are typical for dSphs, mostly populated with old red giant branch (RGB) stars. The red giant branch of KDG61 is situated at the magnitude $F 814 W \geqslant 23.8$. The mean colour of the RGB is $(F 606 W-F 814 W) \simeq 1.1$ at the absolute magnitude level $M_{I}=-3.5$ according to our measurements. The colour spread in the red giant branch is not large for this galaxy, indicating little or no scatter in metallicity. Other prominent feature in the CMD of KDG 61 can be seen above 

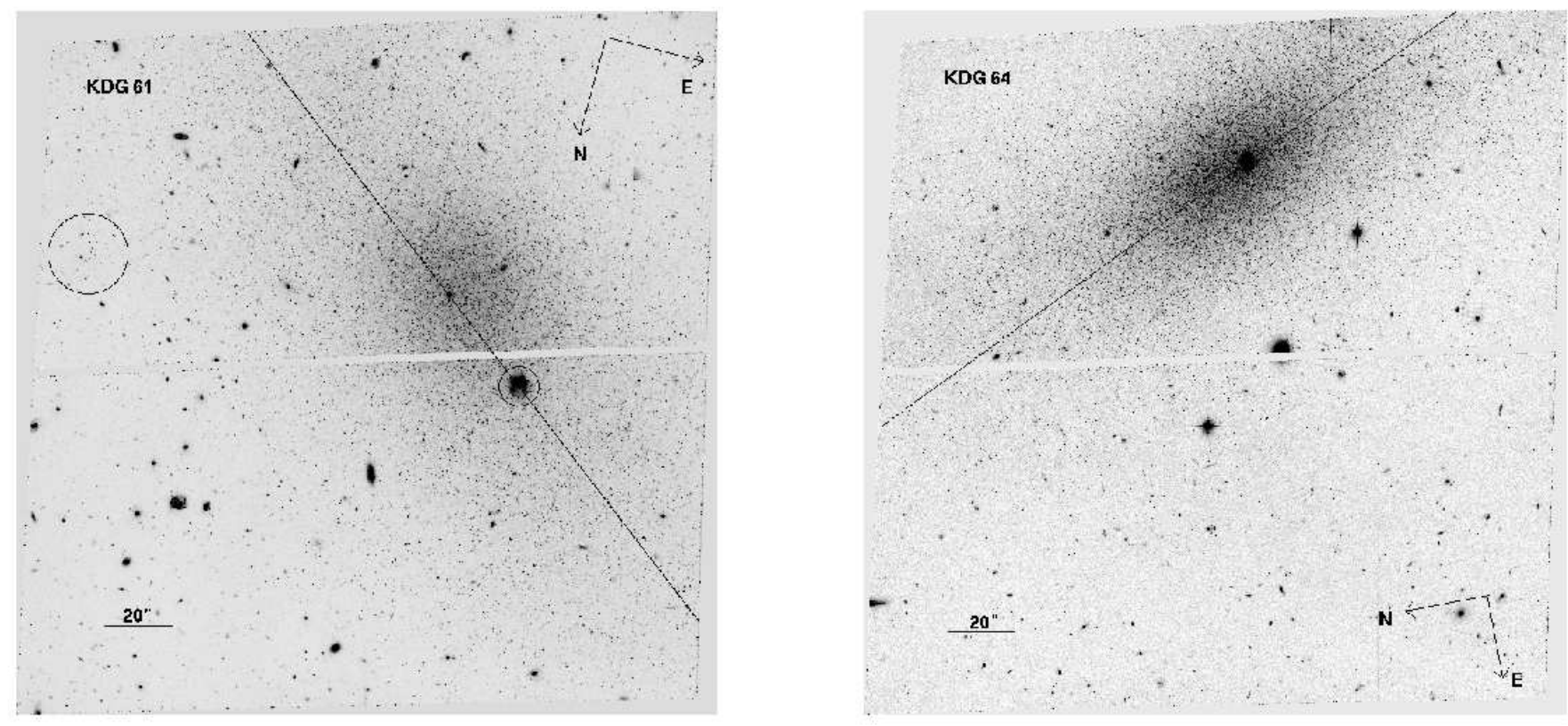

Figure 1. HST/ACS images of KDG 61 and KDG 64 in F606W filter. The position of the spectrograph slit is indicated with line. The slit width is 1 arcsec. The Hil region and the loose concentration of blue stars are indicated with circles (see Sect. 2.2.2).
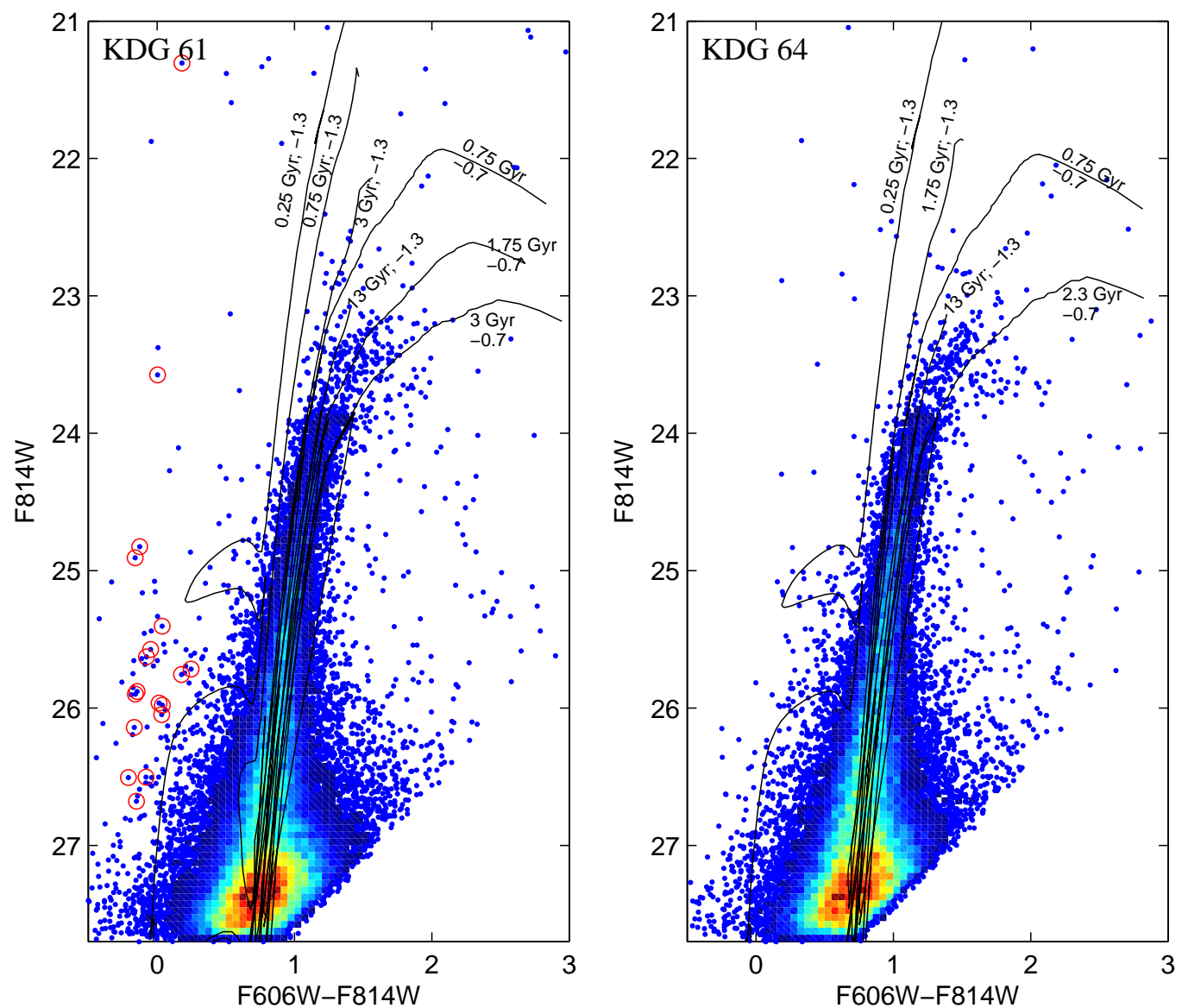

Figure 2. The $(F 606 W-F 814 W), F 814 W$ CMDs of the dwarf galaxies KDG61 (left) and KDG64 (right). In the dense parts of the diagram the colour codes the density in the Hess diagram, while individual stars are represented where they can be individually distinguished. The Padova theoterical isochrones (Girardi et al. 2000) corresponding to the mean age and metallicity of detected star formation episodes were overplotted. The isochrones are labelled with the age in Gyr and with the [Fe/H] metallicity. The magnitudes are not corrected for Galactic extinction. Blue stars resolved in the Hil region of KDG 61 are marked with open circles. 
Table 1. General parameters of KDG 61 and KDG 64

\begin{tabular}{lcc}
\hline & KDG 61 & KDG 64 \\
Position (J2000) & $095703.1+683531$ & $100701.7+674938$ \\
$E(B-V), \mathrm{mag}$ & 0.072 & 0.054 \\
$B_{T}^{0}, \mathrm{mag}$ & 14.93 & 15.29 \\
Axial ratio $b / a$ & 0.58 & 0.47 \\
$(B-R)_{T}^{0}, \mathrm{mag}$ & 1.44 & 1.35 \\
$(V-I)_{e}^{0}, \mathrm{mag}$ & 0.94 & 0.99 \\
Heliocentric velocity, $\mathrm{km} \mathrm{s}^{-1}$ & $221 \pm 3$ & $-15 \pm 13$ \\
Distance modulus $\mu(0), \mathrm{mag}$ & $27.77 \pm 0.04$ & $27.84 \pm 0.04$ \\
Distance, Mpc & $3.58 \pm 0.07$ & $3.70 \pm 0.07$ \\
Spatial separation to M $81, \mathrm{kpc}$ & 40 & 230 \\
$B$ absolute magnitude, mag & -12.84 & -12.55 \\
Central surface brightness in $V$, mag arcsec & -2 \\
Central velocity dispersion, km s & 23.76 & 22.89 \\
Fraction of old stars $(12-14 \mathrm{Gyr})$ & & $25.0 \pm 16.0$ \\
Metallicity of old stars, $[\mathrm{Fe} / \mathrm{H}]$, dex & $82-86 \%$ & $72-89 \%$ \\
\hline
\end{tabular}

the RGB in magnitude range $22.5 \leqslant F 814 W \leqslant 23.8$. These stars likely represent an intermediate age asymptotic giant branch population. There are probably a small number (about 40) of young main sequence blue stars in KDG 61 with a mean colour $(F 606 W-F 814 W) \sim 0.0$, and $23.2 \leqslant F 814 W \leqslant 26.6$. There are also 6 brighter bluish stars in magnitude range of $21 \leqslant F 814 W \leqslant 22$ and colour range of $0 \leqslant(F 606 W-F 814 W) \leqslant 1$. The spatial distribution of the blue stars will be considered in more details below.

The colour-magnitude diagram of the dSph galaxy KDG 64 is similar to the one of KDG61. We can see RGB and intermediate age AGB stars in the same range of magnitudes and colours as in KDG61. However, there are no signs of young blue main sequence at this CMD.

The deep, high-precision photometry of the dSph galaxies allows us to estimate the mean metallicity of the red giant branch using the formula of Lee et al. (1993) : $[\mathrm{Fe} / \mathrm{H}]=$ $-12.64+12.6(V-I)_{-3.5}-3.3\left(V_{I}\right)_{-3.5}^{2}$, where $(V-I)_{-3.5}$ is the mean RGB colour at the absolute magnitude $M_{I}=$ -3.5. According to this equation the mean $[\mathrm{Fe} / \mathrm{H}]$ ratio is equal to $-1.49 \pm 0.03 \mathrm{dex}$ for KDG 61 and $-1.59 \pm 0.02 \mathrm{dex}$ for KDG 64 .

\subsubsection{Foreground contamination}

The CMDs of both KDG 61 and KDG 64 are evidently not heavily contaminated by foreground stars, but as we will perform a detailed analysis of the star formation history, we will make a quantitative assessment of this pollution.

We could not find an appropriate field observed with the HST nearby to the galaxies under study, which would have given us the possibility to estimate a number of foreground stars in these CMDs. An alternative, in this case, is to use simulated star counts in our Galaxy. We have constructed the simulated CMDs of the Galactic stars in the regions of KDG 61 and KDG 64 using TRILEGAL (Girardi et al. 2005). TRILEGAL simulates CMDs in the ACS instrumental system taking into account the components of thin and thick Galactic disks, the halo and the bulge of our Galaxy. The photometric errors, saturation and incompleteness were taken into account in the modelling. These models confirm the negligible contamination by foreground stars. Thus, the expected number of foreground stars in our CMDs would be about 40 up to the photometric limit. We have used also the Besancon model of stellar population synthesis of the Galaxy (Robin et al. 2003) to estimate a number of foreground stars in the fields of KDG 61 and KDG 64. The Besancon model accounts for the four galactic populations: thin and thick disk, spheroid and bulge. The photometric errors were applied to the modelling. The resulting star counts give about 30 stars in our object fields. The synthetic Milky Way stars, according to the TRILEGAL simulations, falls in the magnitude range $20<F 814 W<28$ and the colour range $0.8<F 606 W-F 814 W<2.5$.

In our previous work (Makarova et al. 2002) we already estimated the number of the Milky Way stars in the HST/WFPC2 images of dwarf galaxies in the area of M 81 group. We have analysed observed colour-magnitude diagrams of 24 dwarf galaxies and also theoretical star counts of Bahcall \& Soneira (1981). According to our measurements, we expect between 1 to 5 foreground stars in the magnitude range $18.0 \leqslant I \leqslant 22.0$ and in the colour range $1.4 \leqslant(V-I)_{0} \leqslant 3.0$ in WFPC2 field. This is in a good agreement with the present TRILEGAL simulations, which give us about 4 stars in the same magnitude and colour range, taking into account the field difference between ACS and WFPC2.

\subsubsection{Spatial distribution of the blue stars}

The scarce population of blue stars described above may trace an young star formation event. Therefore, we examine closely their location. We selected blue stars with $(V-I)_{0}<0.4 \mathrm{mag}$ and $I_{0}<26.5 \mathrm{mag}$. In KDG $61,2 / 3$ of the 45 selected blue objects are randomly distributed over the field of the ACS image. The one-sample KolmogorovSmirnov test has shown the homogeneous distribution of the stars. Moreover, the distribution of the blue stars in the ACS field is significantly different from the distribution of all other (red) stars. The two-sample Kolmogorov-Smirnov test rejects the hypothesis of the same distribution of the red and blue stars at the 0.006 significance level, what leads us to the conclusion that the blue stars do not belong to KDG61 itself. Probably, the bluish colour of most of the detected sources is due to a photometric contamination by 

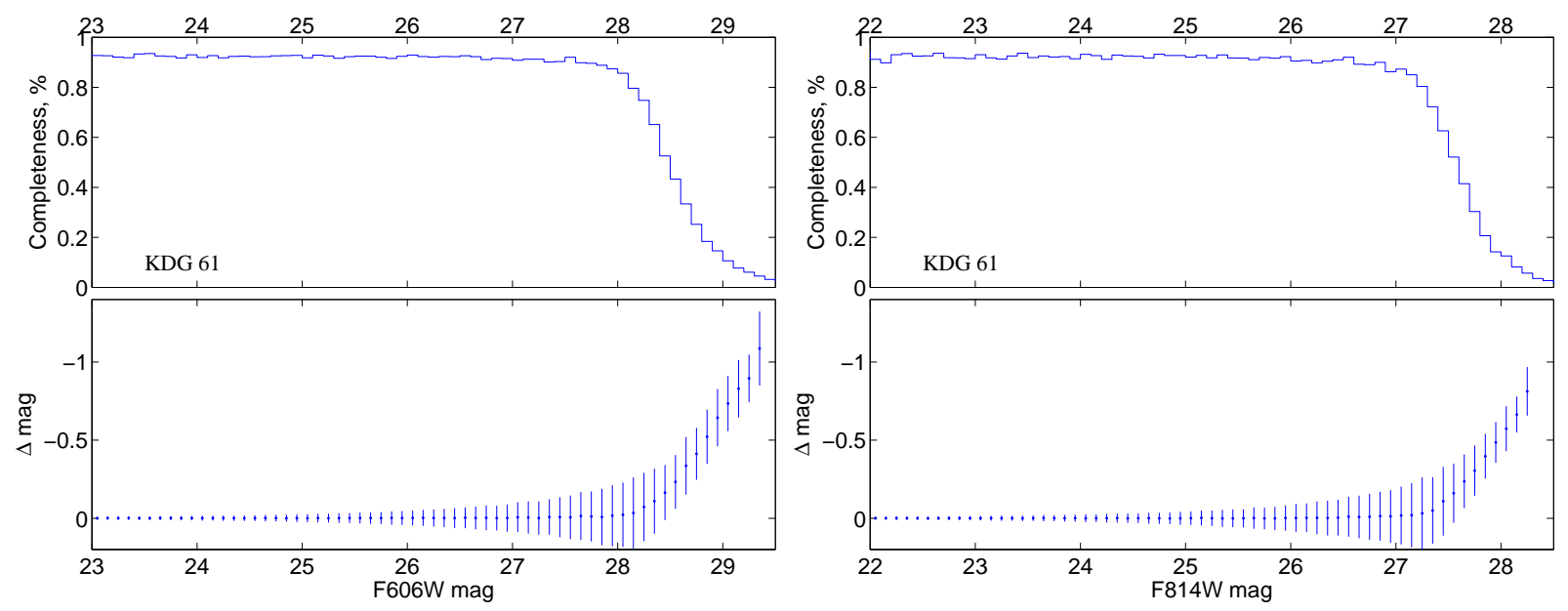

Figure 3. Photometric errors and completeness for KDG61. The top panels show the completeness, i.e. the fraction of artificial stars recovered within the photometric reduction procedure, as a function of the $F 606 W$ and $F 814 W$ magnitudes. The bottom panels give the difference between the measured and the true input magnitude ( $\Delta$ mag $=$ measure - input $)$. The error bars are $1 \sigma$ residuals.

a bright close companion. The rest is associated to the HiI region in KDG 61 and to a small and loose concentration of the bluish stars at the North-West of the galaxy's body. This latter concentration contains only 7 blue stars and we have not enough data to discuss the nature of group of stars associated with it. The two regions are indicated with circles in Fig. 1, and the blue sources associated with the HiI region are marked in Fig. 2.

There is no pronounced blue star population in KDG 64. Dozen of bluish stars, situated outside RGB in the colourmagnitude diagram, are spread over the ACS image and most of them have brighter close companion.

\subsection{Distance determination}

The precise knowledge of the distance is crucial to determine the star formation history from CMD analysis. The photometric tip of the red giant branch (TRGB) distances to the galaxies were previously obtained by Karachentsev et al. (2000) using HST/WFPC2 images. The true distance moduli given in that work are $27.78 \pm 0.15$ for KDG 61 and $27.84 \pm 0.15$ for KDG 64. The corresponding distances are 3.6 Mpc and 3.7 Mpc, respectively. Rekola et al. (2005) has obtained distance moduli of $27.61 \pm 0.17(D=3.3 \mathrm{Mpc})$ for KDG 61 and $27.74 \pm 0.18$ (3.5 Mpc) for KDG 64 using surface brightness fluctuation (SBF) method based on $B$ and $R$-band images obtained with the Nordic Optical Telescope. Both measurements give consistent distances for our galaxies although the SBF measurements seem to give a little shorter scale. However, the difference is inside the error bars.

The present photometry is deeper and it has a higher quality, due to the advantage of the ACS detectors in comparison to the WFPC2. There are also a number of recent improvements implemented to the TRGB method itself. We have determined the photometric TRGB distances with our trgbtool program, which uses a maximum-likelihood algorithm to determine the magnitude of tip of the red giant branch from the stellar luminosity function (Makarov et al. 2006). The measured TRGB magnitudes are $F 814 W_{T R G B}=$
$23.84 \pm 0.02$ for KDG 61 and $F 814 W_{T R G B}=23.87 \pm 0.02$ for KDG 64 in the ACS instrumental system. As pointed in Rizzi et al. (2007), reaching measurements of this quality, the precision on the Galactic extinction becomes a major source of uncertainty. The precision of the extinction on the Schlegel's maps is 16 percents, resulting in an uncertainty of $0.02 \mathrm{mag}$ in the $I$ band. The last source of error is the calibration of the TRGB distance indicator. Using the calibration for the TRGB distance indicator by Rizzi et al. (2007), and adding all the sources or errors, we derived the true distance moduli for KDG 61: $\mu(0)=27.77 \pm 0.04$ $(D=3.58 \pm 0.07 \mathrm{Mpc})$ and KDG 64: $\mu(0)=27.84 \pm 0.04$ $(D=3.70 \pm 0.07 \mathrm{Mpc})$. These new distances are in a good agreement with the previous estimations and they have a better precision.

TRGB distances for several galaxies in M 81 group were recently measured also by Dalcanton et al. (2009) within their ANGST project. The distance moduli of KDG61 (27.72) and KDG 64 (27.85) are in good agreement with our values.

The original Cepheid distance to M 81, 3.63 $\pm 0.34 \mathrm{Mpc}$ (Freedman et al. 1994), was revised to $3.55 \pm 0.13 \mathrm{Mpc}$ (Freedman et al. 2001) $(\mu=27.75 \pm 0.08)$. This value is in close agreement with the TRGB distance from Dalcanton et al. (2009). Recently an independent geometric estimation using the size of the expanding shell around SN1993J gave the distance $3.96 \pm 0.29 \mathrm{Mpc}$ (Bartel et al. 2007). It is also consistent within the uncertainties, but larger than the two other determinations by 10 per cents. Discarding this last value, we adopt a distance $3.58 \pm 0.04 \mathrm{Mpc}$. Beside the value from Dalcanton et al. (2009), the distance to NGC 3077 was determined by the surface brightness fluctuation (Tonry et al. 2001) $\mu(0)=28.03 \pm 0.13$ $(4.04 \pm 0.15 \mathrm{Mpc})$ and TRGB methods (Karachentsev et al. 2003) $\mu(0)=27.91$ (3.82 Mpc). The two TRGB distances agree, and the SBF measurements is larger. We adopt the ANGST value $\mu(0)=27.92 \pm 0.02(3.83 \pm 0.04 \mathrm{Mpc})$

These accurate distances for the two dwarf galaxies allow us to estimate their location within the group of galaxies, and more particularly with respect to M 81 and NGC 3077 . 


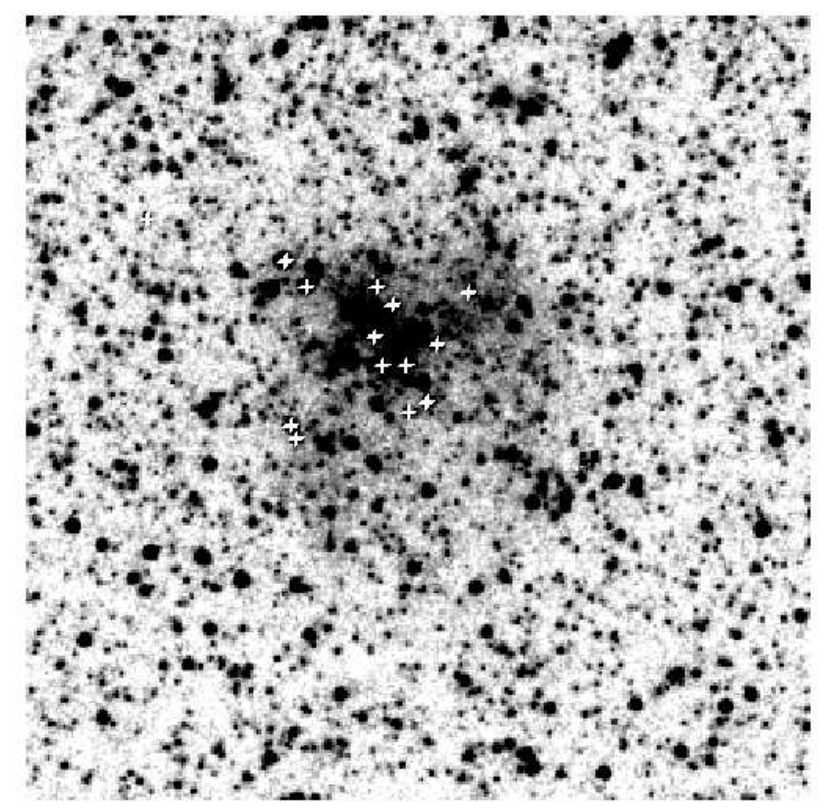

Figure 4. F814W-band image of Hil region in KDG 61. The picture size is $15 \operatorname{arcsec}(250 \mathrm{pc})$. We mark with crosses the resolved blue stars at this region.

Taking into account the distances and angular separation of the objects on the sky, we estimate the spatial separation to M 81 to be about $40 \mathrm{kpc}$ for KDG 61 and about $230 \mathrm{kpc}$ for KDG64. KDG61 is situated very close to the central body of the group. The only galaxies which are likely closer to M 81 are Holmberg IX and BK3N tidal dwarfs. KDG 64 is probably situated on the back side of M 81 and possibly slightly in front of NGC 3077. The spatial separation with NGC 3077 is about $155 \mathrm{kpc}$. Both KDG 61 and 64 are located at the same distance to M 81 as the dSph satellites of the Milky Way (60 kpc to $440 \mathrm{kpc})$.

\subsection{The star formation histories}

The star formation and metal enrichment histories of KDG61 and KDG64 were determined from their CMDs using our StarProbe package (Makarov \& Makarova 2004). This program adjusts the observed photometric distribution of stars in the colour-magnitude diagrams against a positive linear combination of synthetic diagrams of single stellar populations (SSPs, single age and single metallicity). Our approach is similar to the technique described in the works of Tosi et al. (1989), Aparicio et al. (1997) and Dolphin (2000).

The observed data were binned into Hess diagrams, giving the number of stars in cells of the CMDs (twodimensional histograms). The size of the cells are $0.07 \mathrm{mag}$ in luminosity and colour. They are large enough to contain a significant number of stars, and small enough to trace the characteristic features of the CMD distribution. The synthetic Hess diagrams were constructed from theoretical stellar isochrones and initial mass function (IMF). Each isochrone describes the magnitudes and colours of a stellar population with a particular age and metallicity as a map of probabilities to find a star in each cell. We used the Padova2000 set of theoretical isochrones (Girardi et al.
2000), and a Salpeter (1955) IMF. The distance is adopted from the present paper (see above) and the Galactic extinction from Schlegel et al. (1998). The synthetic diagrams were altered by the same incompleteness and crowding effects, and photometric systematics as those determined for the observations using artificial stars experiments.

We have taken into account the presence of unresolved binary stars (binary fraction). As there is no simple and well accepted solution to describe the co-evolution of the close interacting binaries, we used the standard evolution models. The effect of interacting binaries in population models has been found to be small (Zhang et al. 2009). Following Barmina et al. (2002), the binary fraction was taken to be 30 per cents. The mass function of individual stars and the main component of a binary system is supposed to be the same. The mass distribution for second component was taken to be flat in the range 0.7 to 1.0 of the main component mass.

Besides unresolved binary stars a photometric blending (detection of several stars as a single source) should be presented. A blending is sufficient in crowded fields like in globular clusters and in central regions of galaxies. Occasional superposition of stars should distort observational stellar profile. That stars are later excluded by DOLPHOT parameters like sharpness, roundness and characteristic of the PSF-fitting. This effect is taken into account in completeness function constructed from artificial star photometry. However, very close optical binary stars could not be excluded in this way. We have estimated a blending probability for stars brighter than $28.0 \mathrm{mag}$ in $F 814 \mathrm{~W}$ band. For both galaxies it is not exceeded $2 \cdot 10^{-4}$ per cent. Therefore, tacking into account the amount of detected stars the expected number of blended stars is 0.1 over the ACS field. Thus the effect is negligible.

The basis of synthetic diagrams covers all the range of ages (from 0 Myr to $14 \mathrm{Gyr}$ ) and metallicities (from $Z=$ 0.0001 to $Z=0.03$ ), with an age resolution which increases for young populations (2 Gyr steps for the old populations; 
0.5 Gyr near 1 Gyr and younger), and with a metallicity resolution limited by the underlying published isochrones. The isochrones were interpolated in age, to avoid discontinuities, so that the sampled points in the CMD are separated by at maximum 0.03 mag. They were not interpolated in metallicity.

The best fitting combination of synthetic CMDs is a maximum-likelihood solution taking into account the Poisson noise of star counts in the cells of Hess diagram. The resulting star formation history (SFH) are shown in the Fig. 5. The $1 \sigma$ error of each SSP is derived from analysis of likelihood function.

For both galaxies the main star formation event occurred between 12 and 14 Gyr. These initial bursts account for 82 and 89 per cents of the total mass of formed stars for KDG 61 and KDG 64 respectively. The range of metallicity is about $[\mathrm{Fe} / \mathrm{H}]=[-1.6:-1]$ dex. The mean SFR is high in this period for the both objects: $4.510^{-2} \mathrm{M}_{\odot} \mathrm{yr}^{-1}$ for KDG61 and about $5.210^{-2} \mathrm{M}_{\odot} \mathrm{yr}^{-1}$ for KDG 64. These are total star formation rates over the whole galaxy in the ACS image. A rescaling to the total mass of the galaxy would increase the rates by a few percent for KDG 64 .

There are indications of intermediate age star formation in KDG 61 between 2 and 4 Gyr ago and of more recent star formation about $0.5-1 \mathrm{Gyr}$ ago. The measured metallicity range is higher for this period: $[\mathrm{Fe} / \mathrm{H}]=[-1.6:-0.6] \mathrm{dex}$, indicating possible slight metal enrichment for the younger star formation episodes.

We have measured star formation history in two separated regions. For the first sample the stars located within 30 arcsec from the centre of KDG 61 were selected, and for the second sample the outer stars were selected. While the older dominant population is homogeneous, the intermediate age population appears younger (1.5-2 Gyr) in the inner region than in the outskirts (2-4 Gyr). This result is illustrated in the Fig. 6 (two left panels). At the upper panel the SFH in the inner region is shown, and in the lower panel the outer region is shown.

In the case of KDG 64 we detect a slight enhancement of the star formation about 1.5-2.5 Gyr ago and a very small fraction of younger population can have an age of about 500 Myr. A possible metal enrichment over the galaxy lifetime, similar to KDG61, can be present. As for the previous galaxy, we have measured the star formation history of the two separated samples of the resolved stars (inside and outside 30 arcsec). A marginal sign of spatial separation of the stars with different ages was found: The stars of 1.5-2 Gyr dominate in the inner part and stars of 2-2.5 Gyr in the outer part (see Fig. 6). As in KDG61, the older stars are homogeneously distributed. The metallicity of the star formation episodes did not show sufficient differences in the inner regions in comparison to the outer region in the both galaxies.

\section{INTEGRATED LIGHT SPECTRA}

We use the integrated light spectra of KDG 64 and KDG 61 to determine the characteristics of the stellar population. The star formation history reconstruction is affected by the well known age-metallicity degeneracy. This is true for colour-magnitude diagrams as well as for integrated light spectra. Simultaneous usage of the both methods, for the galaxies under study, gives us a hope to improve the reliability of age and metallicity determination.

\subsection{Observations and data reduction}

We obtained spectra of KDG64 with the SCORPIO (Afanasiev \& Moiseev 2005) focal reducer of the 6-meter telescope of the Special Astrophysical Observatory of the Russian Academy of Sciences. The observations were performed in long-slit mode during dark time with $2 \mathrm{k} \times 2 \mathrm{k} C \mathrm{CD}$ EEV42-40 detector (2005 Oct. 6th; PI Philippe Prugniel; Observers Victor Afanasiev and Olga Sil'chenko). We used the 2300 lines per $\mathrm{mm}$ grism, which together with a slit width of 1 arcsec provides a spectral resolution of $2.2 \AA$ (FWHM, corresponding to an instrumental velocity dispersion $\sigma_{\text {ins }} \simeq 56 \mathrm{kms}^{-1}$ or $R \approx 2300$ ), a dispersion of $0.38 \AA /$ pix and a spectral range of $4700-5500 \AA$. The frames were binned by 2 pixels along the slit direction, resulting in a spatial scale of $0.357 \mathrm{arcsec} / \mathrm{pix}$. The observations were made along the position angle, $\mathrm{PA}=24^{\circ}$, corresponding to the major axis of the galaxy. We obtained 9 spectra, each of 20 minutes, resulting in 3 hours in total. We performed arclamp (He-Ne-Ar) calibration at every hour, as well as in the beginning and the end of the night. Thus, we improve the quality of the wavelength calibration, accounting for possible drift due to flexures. Twilight spectra were taken at the beginnings of each night. We used them to correct the variation of the illumination along the slit and to determine the line-spread function of the system. The seeing during these observations was $4.1-4.6$ arcsec.

KDG 61 was observed on 2007 April 17th and 21st with the same observational set-up. The spectra taken during the first night were not used, because of a low quality. Thus, we were left with six exposures of $1200 \mathrm{sec}$ (2 hours). The slit was oriented along the major axis $\left(\mathrm{PA}=54^{\circ}\right)$. It corresponds to the line joining the central cluster with the HıI region.

We attempted to use the spectra obtained by Sharina et al. (2001 on January 18 and 23 2001) with the UAGS longslit spectrograph at the same telescope. Despite comparable exposure times, these spectra have lower $\mathrm{S} / \mathrm{N}$ due to the less efficient instrument and detector and are not useful to complete our data.

The reduction was made in the ESO-MIDAS package with the LONG context. It includes bias subtraction, flat field correction and wavelength calibration. The most critical part of the data reduction is the sky subtraction, as the brightness in the centre of these galaxies is of the order of 10-20 per cents of the brightness of the sky. The main difficulty is the change of the line-spread function (or spectral PSF; hereafter LSF) with the position along the slit. The LSF is the imprint of a spectrograph resolution plus the data reduction (imperfect wavelength calibration). A simple interpolation between two sky regions as proposed in MIDAS is not appropriate because the LSF is narrower in the centre where the galaxy is placed than in the edges where the sky can be estimated. This pattern is however not symmetrical, and we extracted the sky from a region of the slit were the LSF is almost similar to the one in the galaxy region. We determined the relative LSF between the sky and 

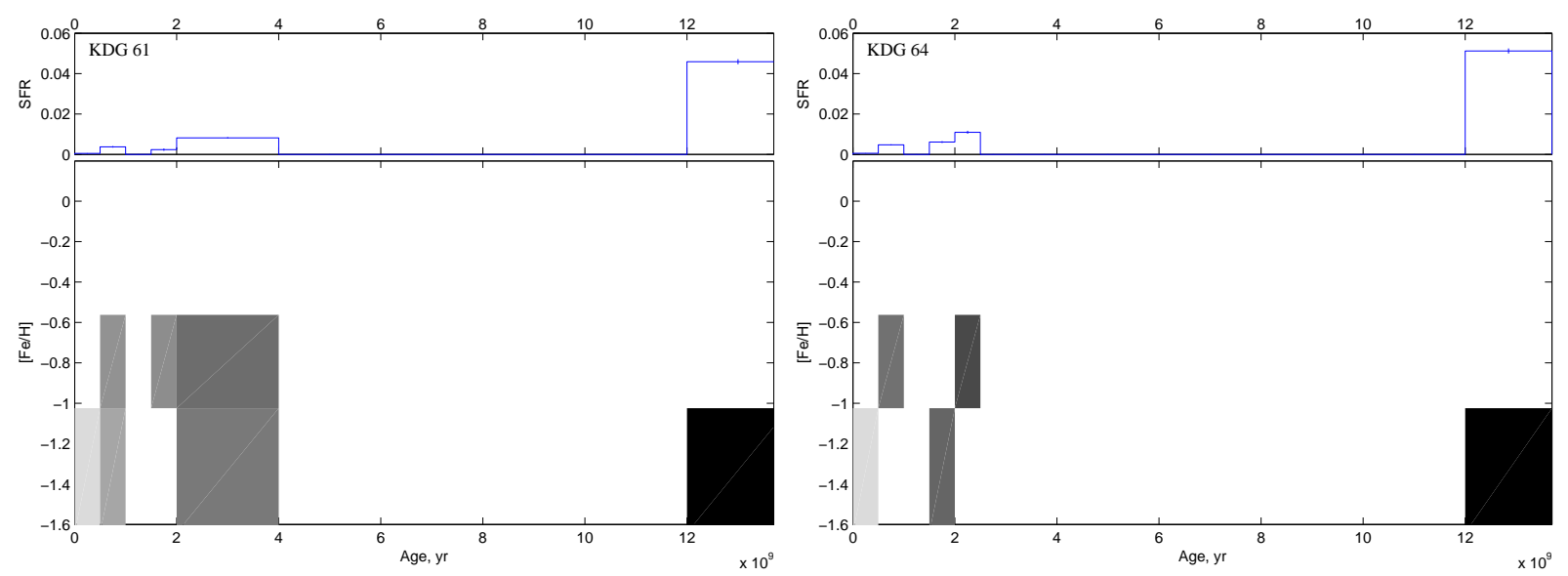

Figure 5. The star formation histories of the dwarf galaxies KDG 61 (left) and KDG 64 (right). Top panels show the star formation rate (SFR) $\left(M_{\odot} / \mathrm{yr}\right)$ against the age of the stellar populations. The bottom panels represent the metallicity of stellar content as function of age. The grayscale colour corresponds to the strength of SFR for given age and metallicity.
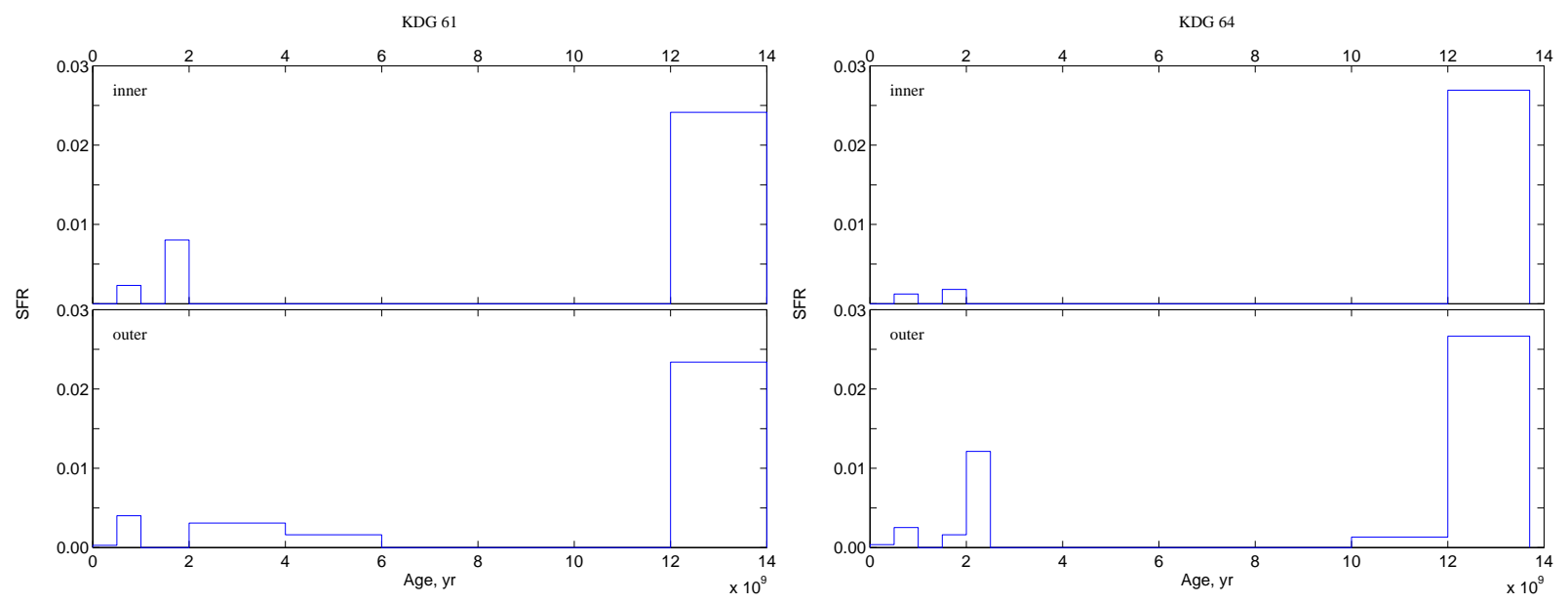

Figure 6. The star formation histories of the dwarf galaxies KDG 61 (left) and KDG 64 (right) in the inner and outer regions. All panels show the star formation rate $(\mathrm{SFR})\left(M_{\odot} / \mathrm{yr}\right)$ against the age of the stellar populations.

object extractions (see Koleva et al. 2009b) and injected it in the galaxy spectrum before subtracting the sky.

There is a significant $\mathrm{H}_{\beta}$ emission line in the sky. It is partly due to the geocoronal fluorescence emission of the atomic Hydrogen from the thermosphere and exosphere ionized by the solar radiation at altitudes between 500 and $80000 \mathrm{~km}$ (Sahan et al. 2007) and partly due to a possible Galactic contribution. As the redshift of our targets are nearly zero, the residuals of the subtraction of this component can alter the centre of $\mathrm{H}_{\beta}$.

\subsection{Analysis method}

We use ULySS $^{3}$ (Koleva et al. 2009b) to analyse the longslit spectra. This program minimizes the $\chi^{2}$ between the observations and a combination of SSP models, to fit the characteristics of the population and the line-of-sight velocity distribution (LOSVD) at the same time. The program

\footnotetext{
${ }^{3}$ http://ulyss.univ-lyon1.fr
}

uses all the pixels of the spectrum and optimally exploits the available information. It fits the observation data as

$$
\begin{aligned}
\operatorname{Obs}(\lambda)=P_{n}(\lambda) \times & \operatorname{LOSVD}\left(v_{\text {sys }}, \sigma\right) \otimes \\
& \sum_{i=0}^{i=m} W_{i} S S P_{i}(\text { Age },[\mathrm{Fe} / \mathrm{H}], \lambda) .
\end{aligned}
$$

The model used in this paper may be either a single SSP or a combination of several SSPs parametrized by their age and metallicity $([\mathrm{Fe} / \mathrm{H}]) . W_{i}$ are the weights of each of the SSPs. The SSPs are convolved by the LOSVD which is parametrized by the systemic velocity $\left(v_{\text {sys }}\right)$ and the velocity dispersion $(\sigma)$. A multiplicative polynomial of order $n, P_{n}(\lambda)$, renders the method insensitive to the extinction an imperfectness in the flux calibration. For this paper we find $n=20$ to be the optimal order of the polynomial. More details on the analysis method can be found in Koleva et al. (2009b). The retrieved solution and errors are checked via $\chi^{2}$-convergence map and Monte Carlo simulations. The full spectrum fitting was extensively validated with Galactic globular clusters in Koleva et al. (2008) and was used to study dE galaxies in Koleva et al. (2009a). 


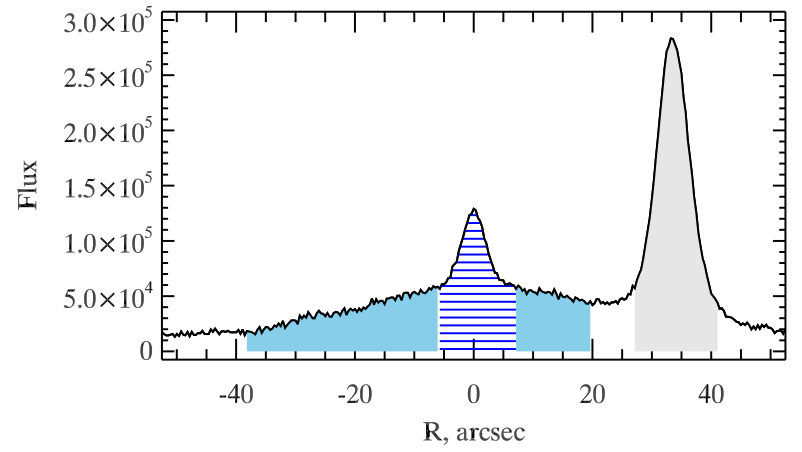

Figure 7. Luminosity profile of KDG 61. The grey shadowed area, centred at about 35 arcsec, shows the extracted region representing the Hil knot. The blue shadowed area is the galaxy's region. The horizontal blue lines mark the globular cluster region.

Our SSPs spectra are computed with Pegase.HR code (Le Borgne et al. 2004) using Padova1994 isochrones (Bressan et al. 1993, and companion papers), Salpeter's IMF (Salpeter 1955) and Elodie.3.1 stellar library (Prugniel \& Soubiran 2001; Prugniel et al. 2007).

\subsection{KDG 61}

We could not perform 2D analysis and investigate the stellar population parameters along the galaxy's radius due to low $\mathrm{S} / \mathrm{N}$. However, we could reach $\mathrm{S} / \mathrm{N} \geqslant 5$ binning the spectra in three different regions. The first one is the central part of 14 arcsec containing the globular cluster. The second is the Hir knot. The third is the galaxy itself excluding the previous regions and extended to a diameter of $40 \mathrm{arcsec}$. We did optimal extraction weighting the individual spectra by their errors. The location of these three extractions is presented in Fig.7. This three extractions were analysed separately.

The next step of the analysis is to determine precisely the LSF. We modelled it with a Gaussian plus GaussHermite distortion terms changing with the wavelength and position along the slit. The LSF was injected in the models (see Koleva et al. 2009b) to take into account the variation of the resolution with the wavelength and to correct the small residual errors in the wavelength calibration.

We use the twilight sky to determine the wavelength dependence of the LSF. We also analysed the background sky of the galaxy against high resolution dark sky spectra obtained with UVES (Hanuschik 2003) convolved by $13 \mathrm{~km} \mathrm{~s}^{-1}$ (to match the resolution of our models). We used the dark sky between $4800 \AA$ to $5000 \AA$ to adjust the zero-point of the velocity. These values were consistent between the globular cluster's and galaxy's extractions. In this paper we do not present or discuss the derived physical velocity dispersion, $\sigma$, because it is highly uncertain.

\subsubsection{Hi knot}

We fitted the position, the width and the intensity of [OiII]4958, [Oiri]5007 and $\mathrm{H}_{\beta}$ using ULY_Line. For the three lines we have heliocentric corrected cz of $-126,-126$ and

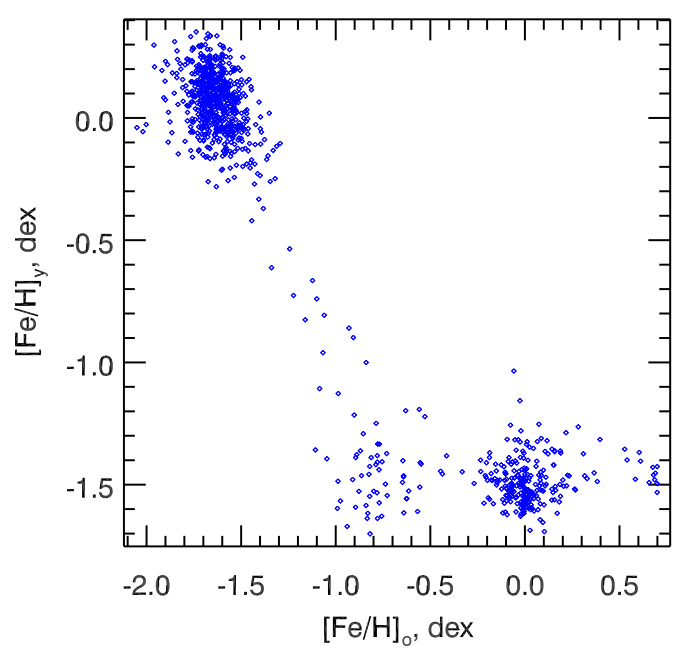

Figure 8. MC simulations for the 2 component fit of K61. In $\mathrm{x}$-axis we plot the metallicity of the old component and in the $\mathrm{y}$-axis the metallicity of the young component.

$-117 \mathrm{~km} \mathrm{~s}^{-1}$ with internal precision of $3 \mathrm{~km} \mathrm{~s}^{-1}$ and systematic uncertainty of $5 \mathrm{~km} \mathrm{~s}^{-1}$ (arising from the determination of the velocity zero-point). We adopt an average $\mathrm{cz}=-123 \pm 6 \mathrm{kms}^{-1}$ which is in agreement with the two previous measurements $-116 \pm 21 \mathrm{~km} \mathrm{~s}^{-1}$ (Sharina et al. 2001) and $-135 \pm 30 \mathrm{~km} \mathrm{~s}^{-1}$ (Johnson et al. 1997).

\subsubsection{Central globular cluster}

The spectrum of the globular cluster was fitted against a grid of SSPs. We obtained heliocentric velocity of $\mathrm{cz}=222 \pm$ $3 \mathrm{kms}$. This velocity is in qualitative agreement with the result from Croxall et al. (2009), showing the $\mathrm{H}_{\beta}$ absorption line of the cluster redshifted with respect to the HiI knot. We find that SSP-equivalent age of the globular cluster is $16 \pm 2 \mathrm{Gyr}$, and its metallicity is $[\mathrm{Fe} / \mathrm{H}]=-1.5 \pm 0.1 \mathrm{dex}$. The errors were determined using Monte Carlo simulations (1000 realizations of the noise).

\subsubsection{Galaxy's body}

We have found that the heliocentric velocity of the galaxy is $\mathrm{cz}=221 \pm 3 \mathrm{~km} \mathrm{~s}^{-1}$. We determined a relatively young SSPequivalent age $1.7 \pm 0.5 \mathrm{Gyr}$ and $[\mathrm{Fe} / \mathrm{H}]=-0.9 \pm 0.2$ dex The errors were determined using 1000 Monte Carlo simulations.

The CMD fit revealed the presence of young stars from 0.5 to 3 Gyr superimposed on the dominant 13 Gyr old population (see Sect. 2.4). The $\mathrm{S} / \mathrm{N}$ of the spectrum is not sufficient to perform an unconstrained analysis of the star formation history as was done in Koleva et al. (2009a). However, bounding the age distribution we may expect to obtain a tight constraint on the metallicity of the two epochs of star formation.

Therefore, we fitted the spectrum with two SSP models fixing the ages to those given by the CMD analysis, i.e. 13 and 0.8 Gyr. It gives us the metallicity and portion of the old component to be $[\mathrm{Fe} / \mathrm{H}]=-1.5 \pm 0.2$ and 86 per cents of the 


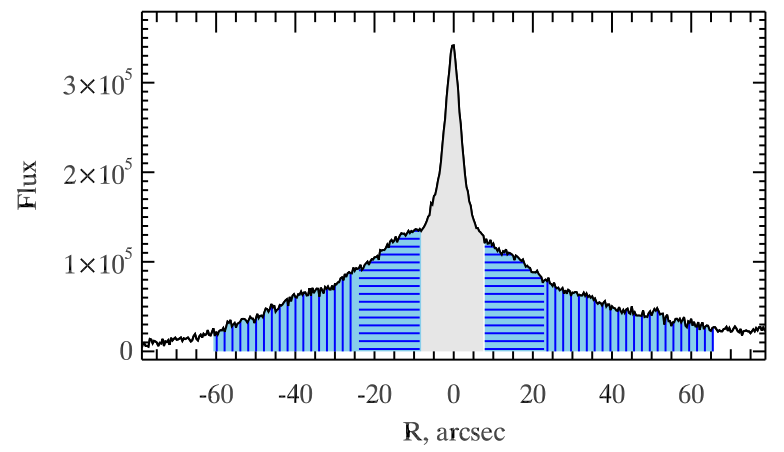

Figure 10. Intensity profile of KDG 64. With grey we mark the extracted region representing the background galaxy (the central 15 arcsec). With blue we show KDG 64 extraction and with dark blue horizontal and vertical lines we show the inner and outer extracted regions, respectively.

stellar mass. The young population comprises 14 per cents of the mass with $[\mathrm{Fe} / \mathrm{H}]=0.0 \pm 0.3$. The error bars were determined using Monte Carlo simulations (Fig. 8). It shows the existence of two solutions. The second one, less populated, has inverse metallicities between the two bursts and is physically less plausible.

The metal enrichment of the young with respect to the old population is stronger than what is suggested by the CMD analysis.

\subsection{KDG 64}

The object in the centre of KDG64 (Binggeli \& Prugniel 1994) is known to be a 1 arcsec diameter background spiral galaxy (Karachentsev et al. 2000; Simien \& Prugniel 2002; Sharina et al. 2001). We have determined the radial velocity of the background galaxy to be $\mathrm{cz}=57540 \mathrm{~km} \mathrm{~s}^{-1}$ and have fitted its stellar population in order to properly model its contamination in the KDG 64 spectrum. See the details of the analysis in Appendix A.

We have extracted the region of the galaxy in a diameter of 110 arcsec excluding the 15 arcsec central region contaminated by the background galaxy (Fig.10). We also split the extraction in inner and outer regions (as it is shown in Fig.10) to search for radial changes in the galaxy's stellar population.

As for KDG 61 (Sect.3.3) we corrected the zero point of the velocity using the UVES sky spectrum.

\subsubsection{SSP-equivalent analysis}

The fit against one SSP shows an age of $4 \pm 2 \mathrm{Gyr}$ and metallicity of $-1.5 \pm 0.1$ dex. For the radial velocity we find $-15 \pm 13 \mathrm{kms}^{-1}$ in agreement with $-18 \pm 14 \mathrm{kms}^{-1}$ from previous measurements of Simien \& Prugniel (2002).

\subsubsection{Star formation history}

For multi-population analysis we tried to decompose the spectrum in two components: young $(<2.5 \mathrm{Gyr})$ and old fixed at 10 Gyr. This choice for the old population gives smaller residuals than using the age of 13 Gyr inferred from the CMD analysis. We found the young component to have an age of $\sim 1.6 \pm 1 \mathrm{Gyr}$ which is consistent with the CMD studies. However, the mass fraction in the young component is 38 per cents, which is significantly higher than indicated from the CMD analysis (11 per cents). To determine the values and the errors of the metallicity we performed 1000 Monte-Carlo simulations. The metallicity of the old component is $[\mathrm{Fe} / \mathrm{H}]=-1.6 \pm 0.4 \mathrm{dex}$. The young stars have almost the same metallicity $[\mathrm{Fe} / \mathrm{H}]=-1.5 \pm 0.4$ dex. Therefore the galaxy shows no metallicity evolution. This result is consistent with the CMD analysis.

\subsubsection{Radial profiles of the population}

Encouraged from the good results and the higher $\mathrm{S} / \mathrm{N}(\sim 8)$ comparable to KDG 61, we tried to find population gradients along the galaxy. To be consistent with the CMD studies we compared the inner 30 arcsec part and the rest of the galaxy. We could not find any difference in the two extractions. The ages and metallicities are the same within the error bars like the general extraction.

\section{DISCUSSION}

\subsection{The nature of the HII knot in KDG 61}

According to our measurements presented above, there is a redshift difference of $344 \pm 6 \mathrm{~km} \mathrm{~s}^{-1}$ between the stellar population of the galaxy and the Hir knot. This knot $\left(\mathrm{cz}=-123 \mathrm{~km} \mathrm{~s}^{-1}\right)$ is associated with the Hi cloud detected at the position of the galaxy (van der Hulst 1979; Boyce et al. 2001, cz $=-140 \mathrm{~km} \mathrm{~s}^{-1}$ ). Thus, because the velocity difference is one order of magnitude larger than the expected stellar velocity dispersion, we can conclude that the gas is not gravitationally bound to the main body of KDG 61.

KDG 61 is spatially close to M 81 . We estimated its separation to be about $40 \mathrm{kpc}$. The velocity difference with $\mathrm{M} 81$ is $258 \pm 4$, adopting $\mathrm{cz}=-37 \pm 3 \mathrm{~km} \mathrm{~s}^{-1}$ for M 81 (Paturel et al. 2003, HyperLEDA). Therefore, KDG 61 is probably near the closest approach on an orbit highly inclined on the plane of the sky. Such a pass could strip the gas from the galaxy, but it is very unlikely that the Hi cloud and His knot were bound to KDG 61 in the past. It is well known that the central part of the M 81 group is embedded into the huge $\mathrm{HI}$ cloud associated with the interaction between M 81, M 82 and NGC 3077 galaxies. Thus, the most likely hypothesis is an alignment between the gaseous and stellar structures on line of sight.

Johnson et al. (1997) discussed the nature of this emission region and stressed its peculiarity. The ratio $[\mathrm{OIII}] 5007 / \mathrm{H}_{\beta}$ is as high as in AGN and $[\mathrm{NII}] 6584 / \mathrm{H}_{\alpha}$. Thus, it appears to be in the transition region between HII regions and AGNs on the Baldwin et al. (1981) diagnostic diagram. Johnson et al. (1997) derived a metallicity of about 0.1 of the solar. It is confirmed recently by Croxall et al. (2009). Assuming that this gas belongs to the galaxy, the system would lie far out from the metallicity versus luminosity relation. The oxygen abundance is too high for such a low mass object and it is close to that of M 81. This characteristic 


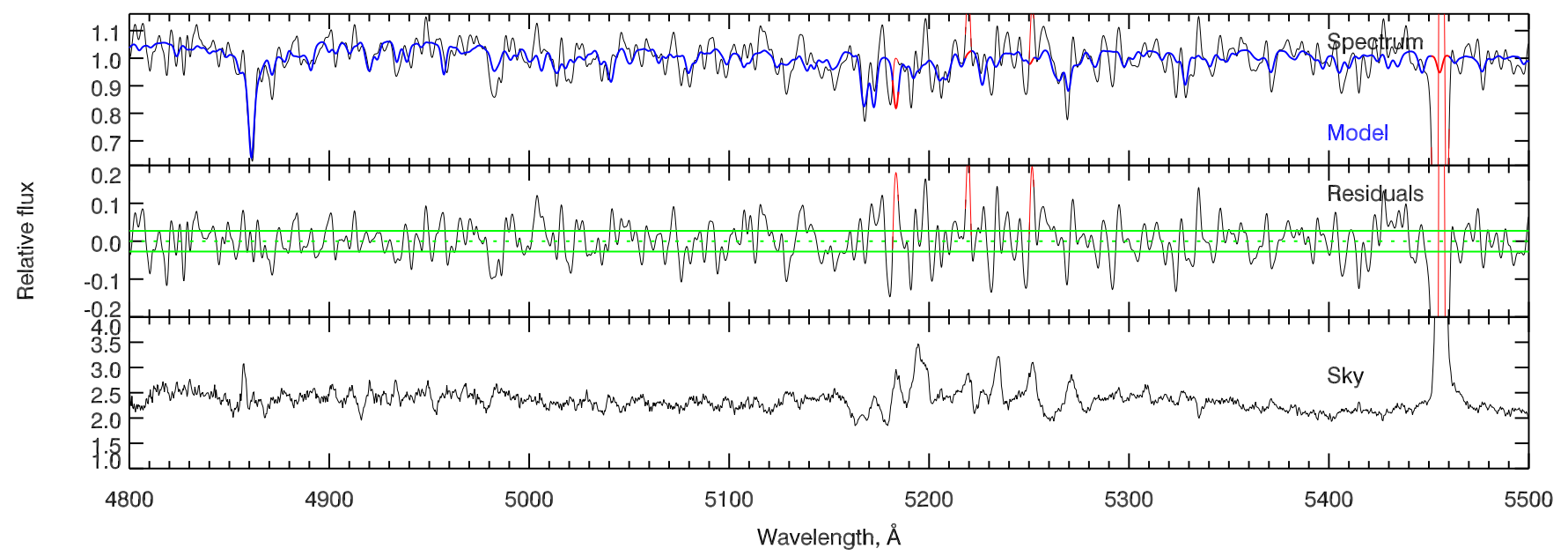

Figure 9. A SSP fit of the central globular cluster of KDG61. From top to the bottom: with black we plot the spectrum and with blue the best fit model; the residuals from the fit (note the extended scale) and the $1 \sigma$ error (in green); the sky extracted from the edge of the $2 \mathrm{D}$ image. In all the panels the masked pixels are plotted with red.

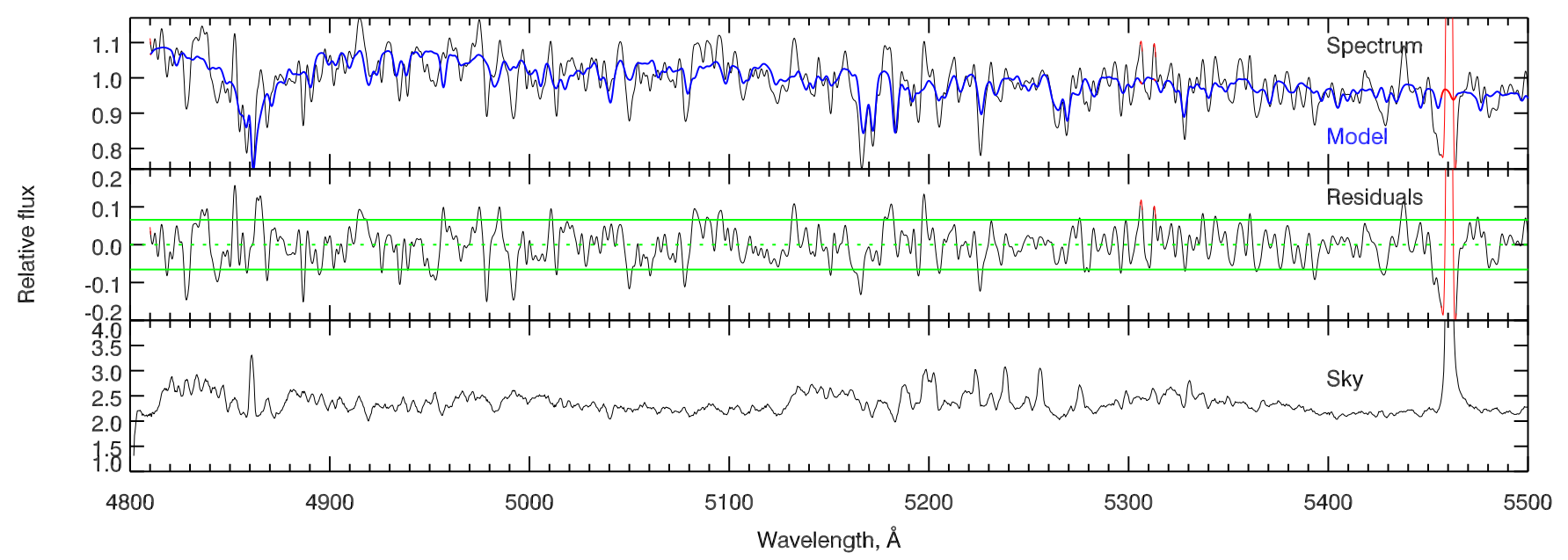

Figure 11. Two component fit of KDG64. In the upper panel with black we plot the observations, smoothed with a Gaussian with a $\sigma=40 \mathrm{~km} \mathrm{~s}^{-1}$, with blue we plot the best fitted model. In the middle panel we show the residuals (with black), smoothed in same way as the observations, the one sigma error is in green. The masked pixels are with red. In the last panel we show the sky.

is shared by two other small objects of the group: Garland and Holmberg IX. Moreover, these objects lie in the regions of the Hi streams connecting M 81, M 82 and NGC 3077. All these facts lead the authors to suggest that Garland, Holmberg IX and KDG 61 are recently formed tidal dwarfs (Holmberg IX and Garland were already considered as tidal dwarf galaxies by van Driel et al. 1998; Makarova et al. 2002; Boyce et al. 2001). However, unlike other proposed tidal dwarfs (as Holmberg IX, see Sabbi et al. 2008) KDG 61 does contain a dominant old population. In the Arp's loop, de Mello et al. (2008) interpret the intermediate-age population as an ejection from the disk of M82 and M81 after the encounter. However, such an interpretation would not hold for KDG 61, as the old population does not resemble a disk population.
The tidal origin of KDG 61 is confidently ruled out, and this galaxy appears to be a classical, bona-fide dSph.

Croxall et al. (2009) also examines various possibilities for the nature of this Hil knot. They rule out the cases of a supernova remnant ([SiII $] 6718 / \mathrm{H}_{\beta}$ is too low) or planetary nebula (too extended). The hypothesis of a wind blown superbubble is the most likely. The detection of [HeII]4686 in the spectrum may be the signature of an ionization by Wolf Rayet stars. The horseshoe morphology of the region at the ACS image, the presence of several blue stars $(\approx 10 \mathrm{Myr}$, $\mathrm{OB}$ association) and the size of the region (major axis of 90 pc) strengthen this hypothesis. The magnitudes integrated in a 4.5 arcsec aperture $(80 \mathrm{pc}$ ) (corrected for Galactic extinction) are: $V_{0}=18.39 \pm 0.09, I_{0}=18.96 \pm 0.07$ and 
$(V-I)_{0}=-0.57 \pm 0.11$. The dereddened Galex magnitudes for the Hir knot are $m_{\mathrm{NUV}}^{0}=19.11$ and $m_{\mathrm{FUV}}^{0}=19.15$ (we used the reddening coefficients from Seibert et al. 2005), similar to the luminosity of the star forming complex observed in the Hi bridge between M 81 and M 82 (Arp's loop) by de Mello et al. (2008).

\subsection{The nature of KDG 61 and KDG 64}

The environment is often proposed to be responsible for the evolution of the gas-rich small galaxies into $\mathrm{dE} / \mathrm{dSph}$ through a transition-type phase. The M 81 group is an excellent site to catch these phenomena in the act, due to the intense interactions between its members, as seen from the filamentary $\mathrm{HI}$ distribution (Fig. 12). and from the bridges connecting the principal galaxies (van der Hulst 1979; Boyce et al. 2001).

Arguing on the presence of Hi gas, KDG61 was proposed in several occasions to be such a transition type object (Johnson et al. 1997; Karachentsev et al. 2000; Boyce et al. 2001). But as we have seen above, this gas is not related to the stellar population.

Boyce et al. (2001) detected a spur of Hi extending south from NGC 3077 to BK5N and KDG64. The optical position of KDG 64 is close to a peak of Hi (see Fig. 12) with the radial velocity of $-100 \mathrm{~km} \mathrm{~s}^{-1}$ belonging to this feature. The velocity of KDG 64 is $-15 \mathrm{~km} \mathrm{~s}^{-1}$. The separation in velocity between the gas and the stars is not as large as in the case of KDG 61, but a physical association between them is likely not present, too. The velocity difference between KDG 64 and NGC 3077 or M 81 is small $\left(\approx 30 \mathrm{kms}^{-1}\right)$ and the orbit lies probably close to the plan on the sky. We can rule out KDG 64 as being responsible for the tidal spur, which, as suggested by Boyce et al. (2001) may be the result of the interaction between BK5N and NGC 3077.

Only the brightest part of stellar populations $(\sim 3 \mathrm{mag}$ below TRGB) were resolved at the HST/ACS images of KDG 61 and KDG 64. These are numerous, predominantly old (about 12-14 Gyr) red giant branch stars and intermediate age asymptotic giant branch stars, which indicate that star formation episode occurred about 1-4 Gyr ago. At the distance of the M 81 group, the fainter stellar populations, like the horizontal branch and the lower part of the main sequence, are hardly resolved even with very deep exposures at the HST. Therefore, these populations are not seen at the CMDs. Without these details it is difficult to resolve age-metallicity-SFR relation for the oldest $(>6 \div 8$ Gyr) star formation events, due to tight packing of the correspondent isochrones for the brightest part of the CMD. Integrated light spectroscopy brings here more reliable information. In contrast to the M 81 group, dwarf galaxies in the Local Group can be resolved into individual stars including the fainter populations. According to the measurements made by Dolphin et al. (2005), the Local Group dSphs in the same luminosity range as KDG 61 and KDG 64 $\left(M_{B} \sim-11 \div-13 \mathrm{mag}\right)$ reveal a dominant peak of star formation 10-13 Gyr ago with some residual star formation continuing until 1-3 Gyr. It is consistent with our results. A similar star formation history is also found in most of the more massive dwarf galaxies $M_{B} \sim-16$ mag of the sample of Koleva et al. (2009c), but, as variance with the present objects, these galaxies often display strong radial gradients of age and metallicity. It seems that the more luminous systems (2-3 mag brighter) have more resources for a second equally strong enhancing of star formation (about 3-6 Gyr ago). In less luminous systems, according to Dolphin et al. (2005), we can expect one ancient burst of star formation of shorter duration and less metal enrichment.

\section{CONCLUSIONS}

We have derived the star formation histories of two dE/dSph galaxies, KDG 61 and UGC $5442=$ KDG 64, belonging to the nearby M 81 galaxy group.

These galaxies have regular axisymmetric morphologies consistent with an early-type classification. They appear to be dominated by an old stellar population (approximately $12-14 \mathrm{Gyr})$ of low metallicity $([\mathrm{Fe} / \mathrm{H}] \simeq-1.5)$. We also detected stars formed about 1 to $4 \mathrm{Gyr}$ ago in both galaxies with a marginal metal enrichment. KDG 64 is slightly less luminous and it has a higher surface brightness than KDG 61, but both galaxies are nearly a mid point of the luminosity sequence connecting the bright dEs, like, for example NGC 205, and the smaller local dwarfs, like, for example, Sculptor.

The well-known numerical simulations by Yun (1999) gives the time since nearest approach between M 81-M 82NGC 3077 of about 300 Myr ago. Recent star formation events in these and tidal dwarf galaxies agree well with this age. But signs of earlier approaches and, therefore, earlier common star formation events are probably "erased" by the recent interaction, and, therefore, we could not relate the recent star formation event in KDG61 and KDG64 (1-4 Gyr ago) to the large scale star formation event in the M 81 group.

There is no sign of a gaseous component in none of the two galaxies (HI or ionized gas). On the basis of our radial velocity measurements on the stellar components, we reject previous suggestions for $\mathrm{HI}$ clouds association with the dwarfs. We have also found, that the His knot previously suggested to belong to KDG61 is in fact a wind-blown superbubble associated to a Hi tidal stream projected on the line-of-sight.

This study was made with two complementary approaches: CMD fitting and full spectrum fitting. The first method uses CMDs usually obtained with HST/ACS, while the last uses long slit spectra that can be acquired on a ground based telescope. The two methods are consistent and it is notable that the full spectrum fitting can give finer constrains on the metallicity. In full spectrum fitting, the metallicity is directly constrained by the metal absorption lines from the stars, while CMDs rely on broad bands colours and on stellar evolution models. It is the first time that a detailed spectroscopic determination of the $\mathrm{SFH}$ was successfully applied to low surface brightness galaxy (with low $\mathrm{S} / \mathrm{N}$ data).

We like to notice the agreement between the two approaches. It is promising for the future where both methods will be applicable within a distance of $20 \mathrm{Mpc}$. The spectroscopic analysis of low surface brightness objects may also be used, at moderate cost, in a large volume of the local universe to explore the diversity of SFHs, which can be related 


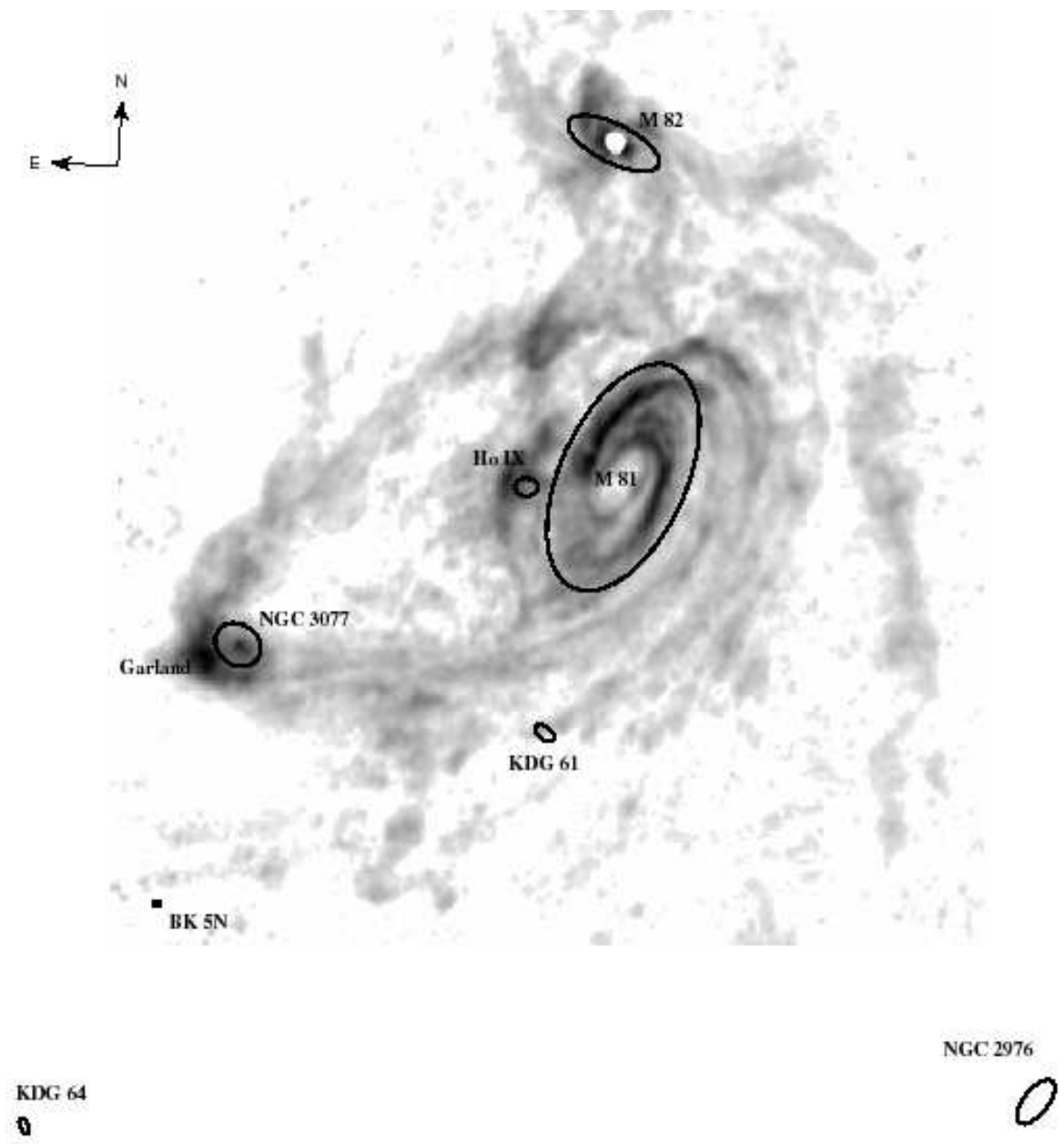

Figure 12. Integrated HI map of the central part of the M 81 group from Yun et al. (Yun et al. 1994). The ellipses indicate the galaxy sizes from Karachentsev et al. (2004).

to other characteristics of the galaxies and to the environment.

\section{ACKNOWLEDGEMENTS}

We are thankful to Olga Sil'chenko and Victor Afanasiev for help with observation. DM, LM and MK thank Observatoire de Lyon for the invitation and hospitality. The work was partially supported by RFBR grants 08-02-00627 and 07-02-00792. MK was supported by DO 02-85/2008 of Bulgarian Scientific Research Fund and Programa Nacional de Astronomía y Astrofísica of the Spanish Ministry of Science and Innovation under grant AYA2007-67752-C03-01.

\section{REFERENCES}

Adami C., Picat J. P., Durret F., Mazure A., Pelló R., West M., 2007, A\&A, 472, 749

Afanasiev V. L., Moiseev A. V., 2005, Astronomy Letters, 31, 194

Aparicio A., Gallart C., Bertelli G., 1997, AJ, 114, 680

Bahcall J. N., Soneira R. M., 1981, ApJS, 47, 357
Baldwin J. A., Phillips M. M., Terlevich R., 1981, PASP, 93,5

Barmina R., Girardi L., Chiosi C., 2002, A\&A, 385, 847

Bartel N., Bietenholz M. F., Rupen M. P., Dwarkadas V. V., 2007, ApJ, 668, 924

Binggeli B., Prugniel P., 1994, La Lettre de l'OHP, 12, 1

Binggeli B., Sandage A., Tammann G. A., 1985, AJ, 90, 1681

Boyce P. J., Minchin R. F., Kilborn V. A., Disney M. J., Lang R. H., Jordan C. A., Grossi M., Lyne A. G., Cohen R. J., Morison I. M., Phillipps S., 2001, ApJ, 560, L127

Bremnes T., Binggeli B., Prugniel P., 1998, A\&AS, 129, 313

Bressan A., Fagotto F., Bertelli G., Chiosi C., 1993, A\&AS, 100,647

Croxall K. V., van Zee L., Lee H., Skillman E. D., Lee J. C., Côté S., Kennicutt R. C., Miller B. W., 2009, ApJ, 705, 723

Dalcanton J. J., Williams B. F., Seth A. C., Dolphin A., Holtzman J., Rosema K., Skillman E. D., Cole A., Girardi L., Gogarten S. M., Karachentsev I. D., Olsen K., Weisz D., Christensen C., Freeman K., Gilbert K., Gallart C., Harris J., Hodge P., de Jong R. S., Karachentseva V., Ma- 
teo M., Stetson P. B., Tavarez M., Zaritsky D., Governato F., Quinn T., 2009, ApJS, 183, 67

de Mello D. F., Smith L. J., Sabbi E., Gallagher J. S., Mountain M., Harbeck D. R., 2008, AJ, 135, 548

de Rijcke S., Penny S. J., Conselice C. J., Valcke S., Held E. V., 2009, MNRAS, 393, 798

De Rijcke S., Prugniel P., Simien F., Dejonghe H., 2006, MNRAS, 369, 1321

Dolphin A. E., 2000, ApJ, 531, 804

-, 2002, MNRAS, 332, 91

Dolphin A. E., Weisz D. R., Skillman E. D., Holtzman J. A., 2005, ArXiv Astrophysics e-prints

Freedman W. L., Hughes S. M., Madore B. F., Mould J. R., Lee M. G., Stetson P., Kennicutt R. C., Turner A., Ferrarese L., Ford H., Graham J. A., Hill R., Hoessel J. G., Huchra J., Illingworth G. D., 1994, ApJ, 427, 628

Freedman W. L., Madore B. F., Gibson B. K., Ferrarese L., Kelson D. D., Sakai S., Mould J. R., Kennicutt Jr. R. C., Ford H. C., Graham J. A., Huchra J. P., Hughes S. M. G., Illingworth G. D., Macri L. M., Stetson P. B., 2001, ApJ, 553,47

Gallart C., Aparicio A., Vilchez J. M., 1996, AJ, 112, 1928

Girardi L., Bressan A., Bertelli G., Chiosi C., 2000, A\&AS, 141,371

Girardi L., Groenewegen M. A. T., Hatziminaoglou E., da Costa L., 2005, A\&A, 436, 895

Grebel E. K., 1999, in IAU Symposium, Vol. 192, The Stellar Content of Local Group Galaxies, Whitelock P., Cannon R., eds., pp. 17-+

Hanuschik R. W., 2003, A\&A, 407, 1157

Irwin M. J., Ferguson A. M. N., Huxor A. P., Tanvir N. R., Ibata R. A., Lewis G. F., 2008, ApJ, 676, L17

Johnson R. A., Lawrence A., Terlevich R., Carter D., 1997, MNRAS, 287, 333

Karachentsev I. D., Karachentseva V. E., Dolphin A. E., Geisler D., Grebel E. K., Guhathakurta P., Hodge P. W., Sarajedini A., Seitzer P., Sharina M. E., 2000, A\&A, 363, 117

Karachentsev I. D., Karachentseva V. E., Huchtmeier W. K., Makarov D. I., 2004, AJ, 127, 2031

Karachentsev I. D., Makarov D. I., Sharina M. E., Dolphin A. E., Grebel E. K., Geisler D., Guhathakurta P., Hodge P. W., Karachentseva V. E., Sarajedini A., Seitzer P., 2003, A\&A, 398, 479

Karachentseva V. E., 1968, Soobshcheniya Byurakanskoj Observatorii Akademiya Nauk Armyanskoj SSR Erevan, 39,62

Koleva M., de Rijcke S., Prugniel P., Zeilinger W. W., Michielsen D., 2009a, MNRAS, 396, 2133

Koleva M., Prugniel P., Bouchard A., Wu Y., 2009b, A\&A, 501, 1269

Koleva M., Prugniel P., De Rijcke S., Zeilinger W. W., Michielsen D., 2009c, Astronomische Nachrichten, 330, 960

Koleva M., Prugniel P., Ocvirk P., Le Borgne D., Soubiran C., 2008, MNRAS, 385, 1998

Koposov S. E., Yoo J., Rix H.-W., Weinberg D. H., Macciò A. V., Escudé J. M., 2009, ApJ, 696, 2179

Le Borgne D., Rocca-Volmerange B., Prugniel P., Lançon A., Fioc M., Soubiran C., 2004, A\&A, 425, 881

Lee M. G., Freedman W. L., Madore B. F., 1993, ApJ, 417, 553
Makarov D., Makarova L., Rizzi L., Tully R. B., Dolphin A. E., Sakai S., Shaya E. J., 2006, AJ, 132, 2729

Makarov D. I., Makarova L. N., 2004, Astrophysics, 47, 229 Makarova L. N., Grebel E. K., Karachentsev I. D., Dolphin A. E., Karachentseva V. E., Sharina M. E., Geisler D., Guhathakurta P., Hodge P. W., Sarajedini A., Seitzer P., 2002, A\&A, 396, 473

Mateo M. L., 1998, ARA\&A, 36, 435

Mieske S., Hilker M., Infante L., Mendes de Oliveira C., 2007, A\&A, 463, 503

Paturel G., Petit C., Prugniel P., Theureau G., Rousseau J., Brouty M., Dubois P., Cambrésy L., 2003, A\&A, 412, 45

Prugniel P., Simien F., 2003, Ap\&SS, 284, 603

Prugniel P., Soubiran C., 2001, A\&A, 369, 1048

Prugniel P., Soubiran C., Koleva M., Le Borgne D., 2007, arXiv:astro-ph/0703658

Rekola R., Jerjen H., Flynn C., 2005, A\&A, 437, 823

Rizzi L., Tully R. B., Makarov D., Makarova L., Dolphin A. E., Sakai S., Shaya E. J., 2007, ApJ, 661, 815

Robin A. C., Reylé C., Derrière S., Picaud S., 2003, A\&A, 409, 523

Sabbi E., Gallagher J. S., Smith L. J., de Mello D. F., Mountain M., 2008, ApJ, 676, L113

Sahan M., Yegingil I., N. A., 2007, Terr. Atmos. Ocean. Sci., 18, 85

Salpeter E. E., 1955, ApJ, 121, 161

Schlegel D. J., Finkbeiner D. P., Davis M., 1998, ApJ, 500, 525

Seibert M., Martin D. C., Heckman T. M., Buat V., Hoopes C., Barlow T., Bianchi L., Byun Y., Donas J., Forster K., Friedman P. G., Jelinsky P., Lee Y., Madore B. F., Malina R., Milliard B., Morrissey P., Neff S., Rich R. M., Schiminovich D., Siegmund O., Small T., Szalay A. S., Welsh B., Wyder T. K., 2005, ApJ, 619, L55

Sharina M. E., Karachentsev I. D., Burenkov A. N., 2001, A\&A, 380, 435

Sharina M. E., Karachentsev I. D., Dolphin A. E., Karachentseva V. E., Tully R. B., Karataeva G. M., Makarov D. I., Makarova L. N., Sakai S., Shaya E. J., Nikolaev E. Y., Kuznetsov A. N., 2008, MNRAS, 384, 1544

Sharina M. E., Puzia T. H., Makarov D. I., 2005, A\&A, 442, 85

Simien F., Prugniel P., 2002, A\&A, 384, 371

Sirianni M., Jee M. J., Benítez N., Blakeslee J. P., Martel A. R., Meurer G., Clampin M., De Marchi G., Ford H. C., Gilliland R., Hartig G. F., Illingworth G. D., Mack J., McCann W. J., 2005, PASP, 117, 1049

Tonry J. L., Dressler A., Blakeslee J. P., Ajhar E. A., Fletcher A. B., Luppino G. A., Metzger M. R., Moore C. B., 2001, ApJ, 546, 681

Tosi M., Greggio L., Focardi P., 1989, Ap\&SS, 156, 295

Trentham N., Hodgkin S., 2002, MNRAS, 333, 423

van der Hulst J. M., 1979, A\&A, 75, 97

van Driel W., Kraan-Korteweg R. C., Binggeli B., Huchtmeier W. K., 1998, A\&AS, 127, 397

Yun M. S., 1999, in IAU Symposium, Vol. 186, Galaxy Interactions at Low and High Redshift, J. E. Barnes \& D. B. Sanders, ed., pp. 81-+

Yun M. S., Ho P. T. P., Lo K. Y., 1994, Nature, 372, 530

Zhang F., Li L., Han Z., 2009, MNRAS, 396, 276 


\section{APPENDIX A: BACKGROUND GALAXY PROJECTED ON KDG 64}

To correct the residual contamination in KDG64 from the background galaxy, we adopted the following strategy:

- We prepared two extractions, one containing mostly KDG 64 (the regions marked with blue horizontal lines on Figure 10) and one containing mostly the background object (the grey part on Figure 10). We named them extraction1 and extraction2, respectively.

- We decomposed the profile along the slit to a Gaussian (for the background galaxy), plus a polynomial (for KDG 64). From this decomposition, we measured that extraction 2 should be contaminated by KDG 64 by 80 per cents. The contamination of the background galaxy in extraction1 should be less than 1 per cent.

- At first we attempted to fit extraction2 with a two component model: one SSP for KDG64 and one SSP for the background galaxy. However, we realized that we cannot fit the background galaxy (extraction2) with a SSP. We adopted a five component model (Fig. A1): 1 SSP for KDG64, 2 SSPs for the background galaxy and 2 delta functions for $\left(H_{\gamma}\right.$ and $\left.H_{\delta}\right)$. We set the limits for the background SSPs to be between 0.001 and $0.5 \mathrm{Gyr}$ and 5 and 16 Gyr. The emission lines were broadened with the LOSVD of the galaxy. We found an young population of $0.016 \mathrm{Gyr}$ with -1 dex of metallicity, weighting 15 per cents in light and 1 percent in mass and an old population of about $7 \mathrm{Gyr}$, super-solar metallicity, 23 per cents in light and 94 per cents in mass (the rest of the light is going to KDG 64 component). Our determination of radial velocity of the background galaxy $\left(\mathrm{cz}=57540 \mathrm{~km} \mathrm{~s}^{-1}\right)$, disagrees with the value published by Sharina et al. $\left(2001, \mathrm{cz}=46530 \mathrm{~km} \mathrm{~s}^{-1}\right)$. After checking the plotted spectrum in Sharina et al. (2001), we conclude that the published value is an error (a typo or wrong conversion). Our estimation of velocity dispersion for the background galaxy is $\sim 130 \mathrm{~km} \mathrm{~s}^{-1}$.

- As a last step we used the best fit of the background galaxy to analyse extraction1. When using single burst decomposition for KDG 64 we found less than 1 per cent of the light going to the background object, consistent with the profile decomposition. 

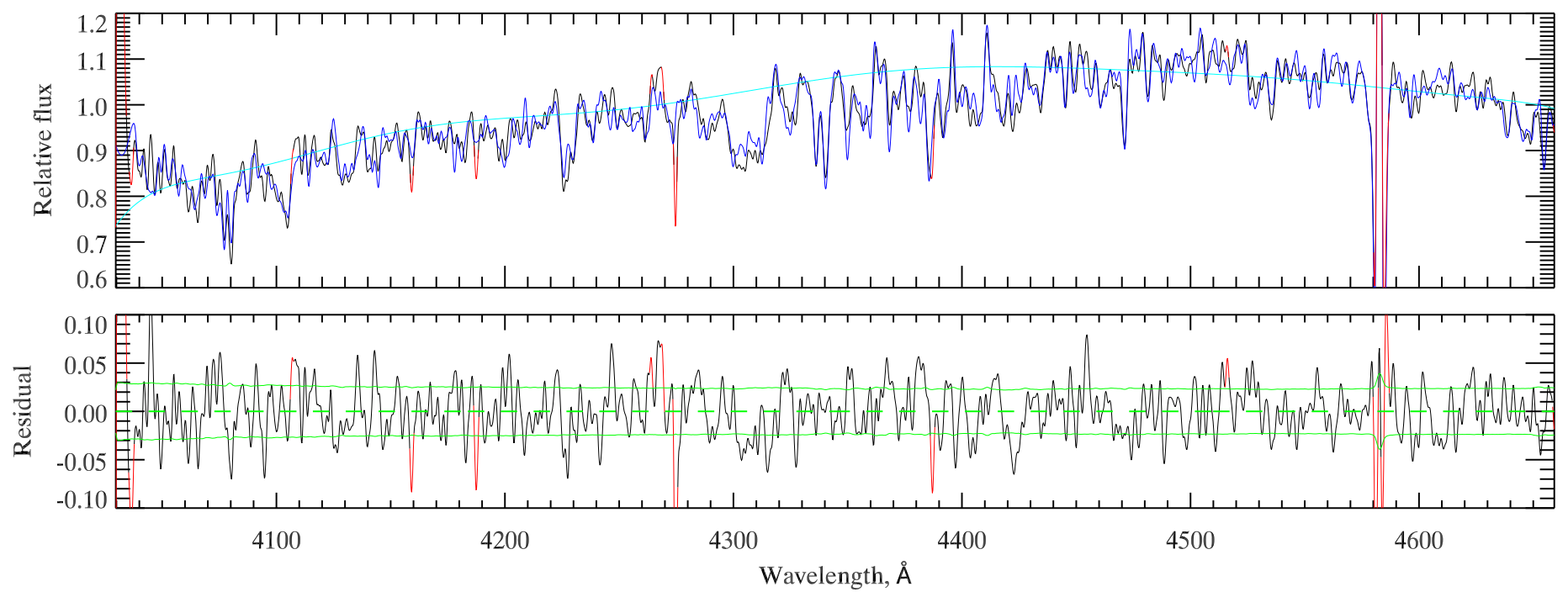

Figure A1. Five component fit (see the text) of the background galaxy. blue is the best fitted model, cyan is the multiplicative polynomial, black the objected smoothed with Gaussian sigma of $40 \mathrm{~km} \mathrm{~s}^{-1}$. In the lower panel we plot the residuals from the fit (in black, a gain smoothed in same way as the observations) and the one sigma error in green. The masked pixels are with red. 